\title{
In-situ Gold-Silicon Eutectic Mixture Formation
}

Daniel Veghte ${ }^{1}$, Carley Goodwin ${ }^{1}$ and James Ranney ${ }^{2}$

${ }^{1}$ The Ohio State University, United States, ${ }^{2}$ Thermo Fisher Scientific, United States

A common way to bond wafer in semiconductors is to use a metal such as gold that forms a eutectic mixture with the silicon wafer. [1] The gold-silicon system can melt at a temperature as low as 363 ${ }^{\circ} \mathrm{C}$ at $18.6 \%$ silicon, much lower than the melting point of gold $\left(1064{ }^{\circ} \mathrm{C}\right)$ or silicon $\left(1414{ }^{\circ} \mathrm{C}\right)$. [2] Previous research has shown that the fraction of silicon is inversely proportional to the bond strength of the material, though higher percentages are common due to processing conditions where silicon is in excess. [3] At high Si concentrations there are large inclusion of silicon that form leading to a lower bonding rate and brittle structures above $57.5 \%$ silicon. [3] This study aims to understand the formation mechanism of the silicon and gold eutectic mixture through using in-situ heating in the environmental scanning electron microscope (ESEM).

In-situ heating experiments on the melting behavior of small sections of gold wire on single crystalline silicon were performed in the Quattro ESEM using the high vacuum heating stage. Heating of the samples near the transitions were performed at $1.5{ }^{\circ} \mathrm{C} / \mathrm{min}$ to observe the phase transitions and determine the melting point. Two different experiments were performed to understand the process of formation and the subsequent product formed. The first experiment determined the melting point of the gold and the resulting melting point of the eutectic mixture formed. The second experiment involved a long gold wire that was only in contact with the silicon wafer at the edges, with the middle elevated. The sample from the second experiment was analyzed using the HeliScan microCT to characterize the diffusion of gold into silicon and vice versa. Data analysis and visualization were performed in the Avizo software suite. Additional analysis was performed by cutting cross-sections in the sample using the Helios NanoLab 600 dualbeam and characterizing the exposed surfaces using the EDAX Octane Elect Super Energy Dispersive X-ray Spectroscopy (EDS) and Velocity Electron Backscatter Diffraction (EBSD) on the Quattro ESEM.

Melting experiments of gold on silicon were performed in the ESEM to observe the structure above and below the melting temperatures. During heating there would often be diffusion of the gold on the silicon surface at temperatures lower than the melting point of gold indicating that diffusion starts to happen at lower temperatures. For experiments where the gold melted before diffusion, the melting point was found to be near $1070{ }^{\circ} \mathrm{C}$ (Figure $1 \mathrm{a}$ a-c), consistent with the its actual melting point (1064 $\left.{ }^{\circ} \mathrm{C}\right)$. Upon cooling of the melted gold, islands of silicon were observed within the gold. When the samples were heated after the initial melting of the gold the gold/silicon would melt and solidify around $470{ }^{\circ} \mathrm{C}$ (Figure $1 \mathrm{~d}$-f). This would be consistent with a gold/silicon eutectic mixture between 17 to 25 atomic percent silicon, near the minimum melting temperature of the $\mathrm{Au} / \mathrm{Si}$ mixture at 363 ${ }^{\circ} \mathrm{C}$ with 18.6 atomic percent silicon. [2] Further analysis of the gold wire sample was performed using microCT to obtain three-dimensional data and observe the interior structure. The microCT data was able to show that the gold diffused into the silicon wafer where it was in contact (Figure 2b). Silicon was also observed in large islands throughout the melted area of the gold, as well as the area suspended above the silicon wafer. This shows diffusion of silicon through gold for tens of microns (Figure $2 \mathrm{c}$ and d). Additionally, a cross section exposed by FIB milling of the sample verified that there were discreet areas of silicon and gold throughout the bulk of the sample (Figure $2 \mathrm{~g}, \mathrm{~h}$ ). Through in-situ 
SEM experiments, we were able to show the process of forming the gold/silicon eutectic mixture through. Analysis of the samples afterwards with microCT and cross-sectional EDS showed the structure of the mixture from the bulk to nanoscale.

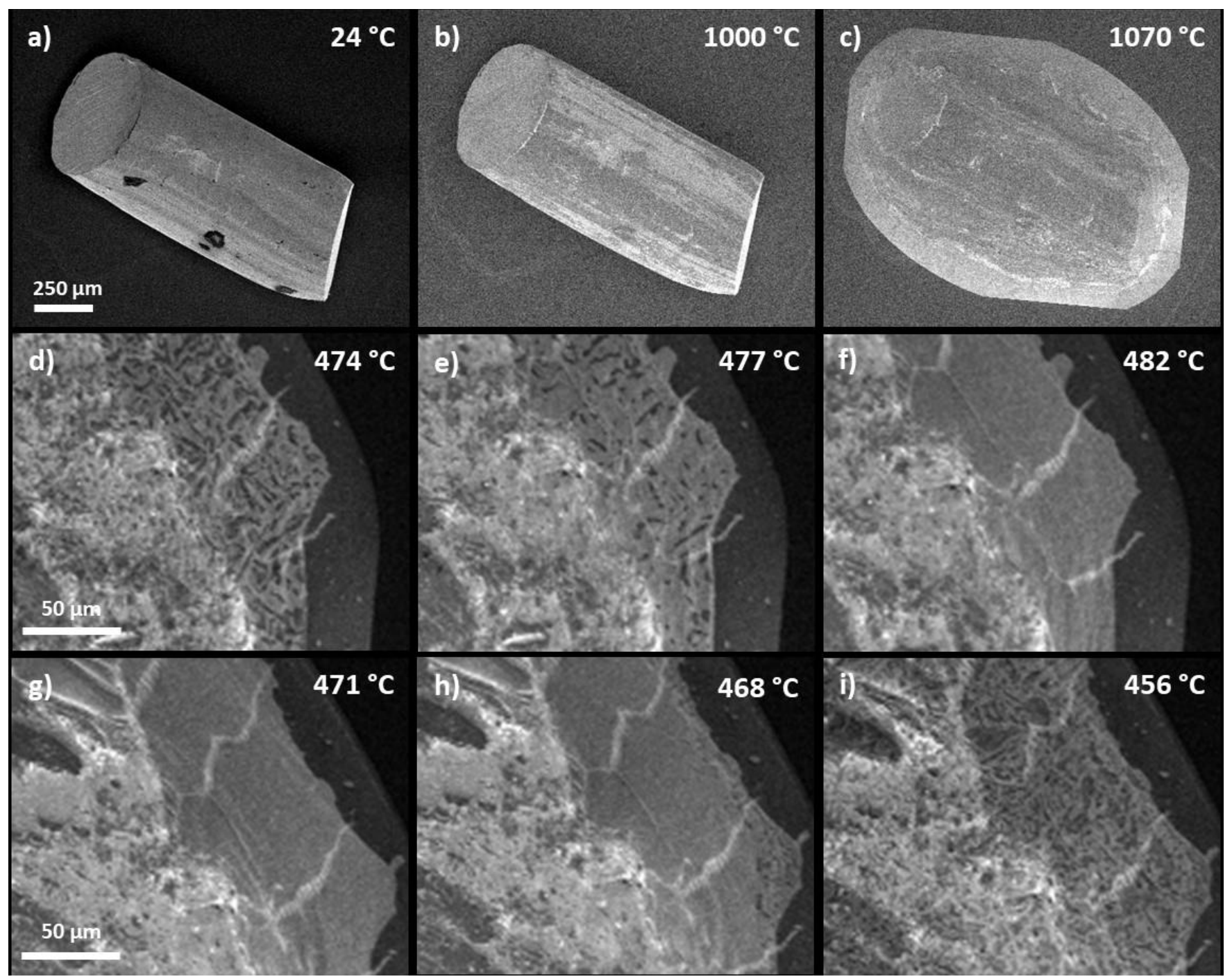

Figure 1. Heating experiment showing a-c) the initial melting of gold by $1070{ }^{\circ} \mathrm{C}$. Subsequent experiments (d-i) with heating and cooling the same sample showed that the gold would melt and solidify at around $470{ }^{\circ} \mathrm{C}$. 


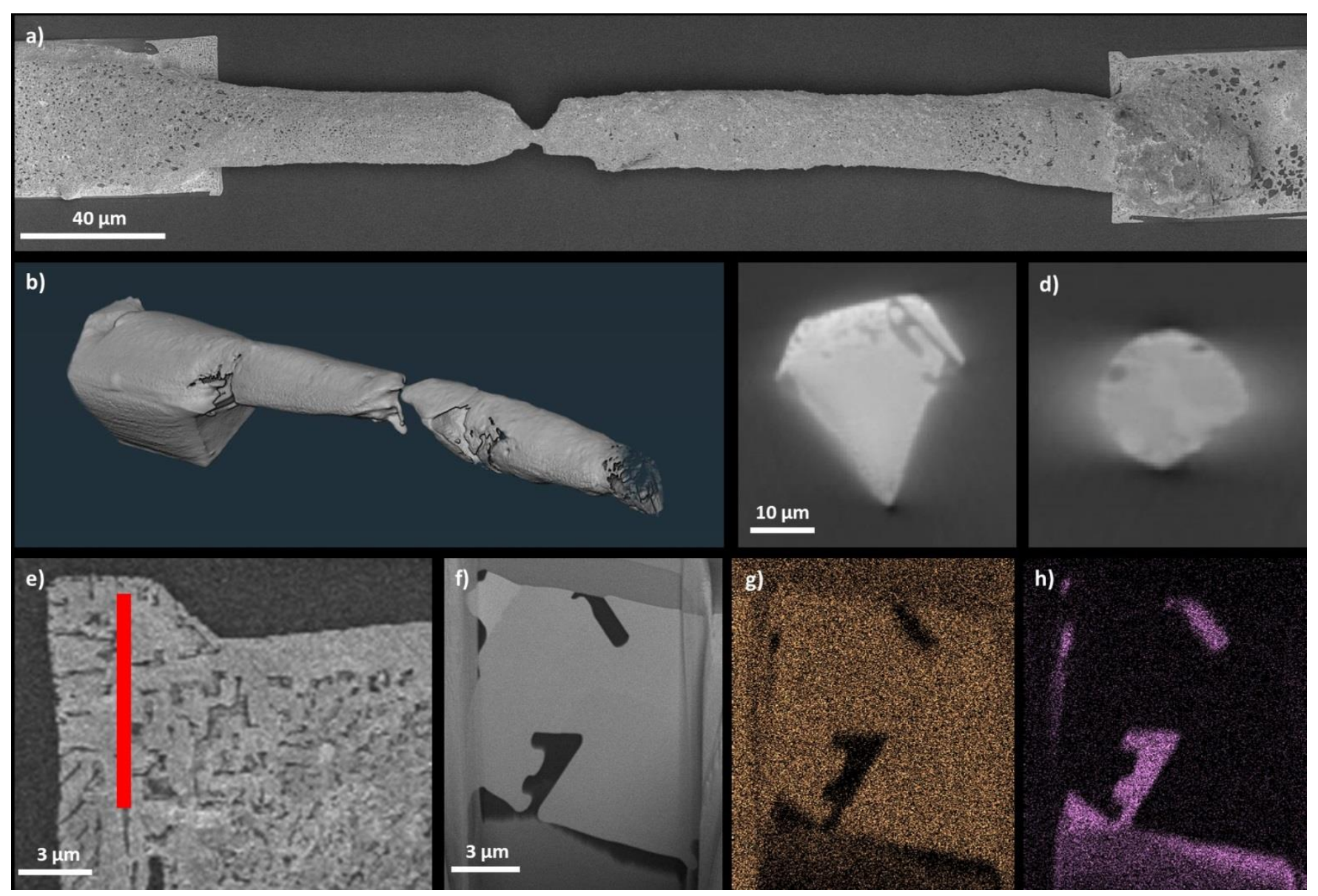

Figure 2. Experiment showing the results after heating a long gold wire where only the ends are in contact with the substrate. a) SEM image of the gold wire where the ends are melted to the substrate and the center has lost volume. b) 3D volume rendering of microCT data of one end of the sample where the gold has diffused into the silicon substrate. c) microCT cross section image of the end and d) elevated area of the gold wire. e) area where the cross section was exposed f) backscatter electron image, g) gold EDS map, and h) silicon EDS map.

\section{References}

[1] Wolffenbuttel and Wise, Sensor and Actuators A, 43, 1994, 223-229

[2] Okamoto and Massalski, Bulitin of Alloy Phase Diagrams, 4,2, 1983, 190-198

[3] Lin et al. 2009, Transducers, 244-247

[4] Electron microscopy and x-ray microCT was performed at the Center for Electron Microscopy and Analysis (CEMAS) at The Ohio State University 